\title{
Therapeutic Management of Theileriosis in Gir Calves: Study of Two Cases
}

\author{
M. J. Bharai ${ }^{1 *}$, T. D. Bhavsar ${ }^{1}$, P. G. Dodiya ${ }^{2}$ and Vijay L. Parmar $^{3}$ \\ ${ }^{1}$ College of veterinary Science and Animal Husbandry, \\ ${ }^{2}$ Polytechnic in Animal Husbandry, \\ ${ }^{3}$ Department of Veterinary Medicine, JAU, Juanagadh-362 001, Gujarat, India \\ *Corresponding author
}

\section{Keywords \\ Buparvaquone, Oxytetracycline, Theileriosis, \\ Article Info \\ Accepted: \\ 04 November 2019 \\ Available Online: \\ 10 December 2019}

\section{Introduction}

Theileriosis is caused by protozoan parasites of the genus Theileria that infect lymphocyte and erythrocytes of ruminants.

The disease is transmitted transstadially by ticks of the genus Ixodidae, causing problem for health and management of domesticated cattle of all ages in tropical and subtropical regions (Morrison, 1998).

A nervous form of the disease, known as cerebral theileriosis, may develop in cattle and caused by Theileria annulata, Theileria parva and Theileria tautragi(Saville, 2002). Present case report deals with successful treatment of cerebral theileriosis in two Gir calves.

\section{Case history and Clinical observations}

Two female cattle calves aged about one year were presented to Department of Veterinary Medicine, TVCC, Junagadh with complaint of anorexia, circling movement, and unable tobear weight properly (Fig. 1).

Clinical examination of the animals revealed severe tick infestation, enlarged prescapular lymph node, depression and hyperaesthesia. Clinical examination depicted pale conjunctival mucous membrane (Fig. 2), pyrexia $\left(102.1^{\circ} \mathrm{F}\right.$ and $\left.103.8^{\circ} \mathrm{F}\right)$, circling movement, weakness and coffee coloured urine. The blood samples with anticoagulant and faecal samples were collected for haematology and microscopic examination. 


\section{Diagnosis}

Haematological examination revealed erythocytopenia with decreased $\mathrm{Hb}, \mathrm{MCV}$ and $\mathrm{MCHC}$ and other parameters viz. PCV, WBC, neutrophils, basophils, eosinophils, lymphocytes and monocytes were within normal range. On microscopic examination of Giemsa stained thin blood smear, schizonts were observed (Fig. 3). Faecal examination showed no helminth eggs or coccidialoocysts.

On the basis of history, physical and clinical examinations and finding of schizonts in blood smears, the cases were diagnosed as cerebral theileriosis.

\section{Treatment and Discussion}

The calves were treated with a single intramuscular injection of Buparvaquone@ $2.5 \mathrm{mg} / \mathrm{kg}$ and oxytetracycline @ $10 \mathrm{mg} / \mathrm{kg}$ intravenously once in a day for three days. This antibiotic is a useful adjunct because in addition to antitheilerial activity (Bagherwal, 1989), it helps to ameliorate the pneumonic changes (Khanna et al., 1983) and it is curative for anaplasmosis which may occur concurrently (Dolan et al., 1992). Plasma extender (Haemaccel ${ }^{\circledR} 3.5 \%$ ), 25\% dextrose and Ringer lactate were administered $\mathrm{i} / \mathrm{v}$ for three days along with Imferon injection $\mathrm{i} / \mathrm{m}$

\section{Fig.1 Theileria affected calves with sternal} recumbuncy

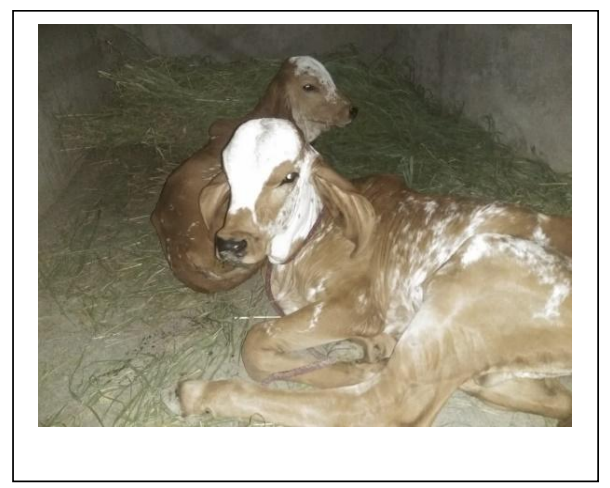

twice a week and liver tonic. The calves were observed for 5 days. The animals made an uneventful recovery on third day of treatment.

Tropical theileriosis commonly presents anaemia in addition to fever, swelling of the lymph nodes and rapid loss of condition. The anaemia that occurs in theileriosis is probably caused by extravascular haemolysis (Lal and Soni, 1985). Although severe anaemia progresses in cattle with theileriosis, unlike in babeiosis, haemoglobinuria rarely occurs (Morrison, loc, cit). In present case, Plasma extender, iron preparation Imferon along with Ringers lactate were administered as supportive therapy for anaemia. Saville (loc. cit) reported that clinical signs of cerebral theileriosis included ataxia, depression, circling movement, head pressing, hyperaesthesia, blindness, hypermetria, nystagmus, proprioceptive deficits and aggressiveness.

When the disease is fatal, the animal become recumbent and develops opisthotonus, tonic and clonic seizures and coma. In this case signs like depression, circling movement, hyperaesthesia and unable to stand up were seen. It was diagnosed early as cerebral theileriosis; therefore cases were treated successfully.

Fig.2 Theileria affected calf with pale conjunctival mucous membrane

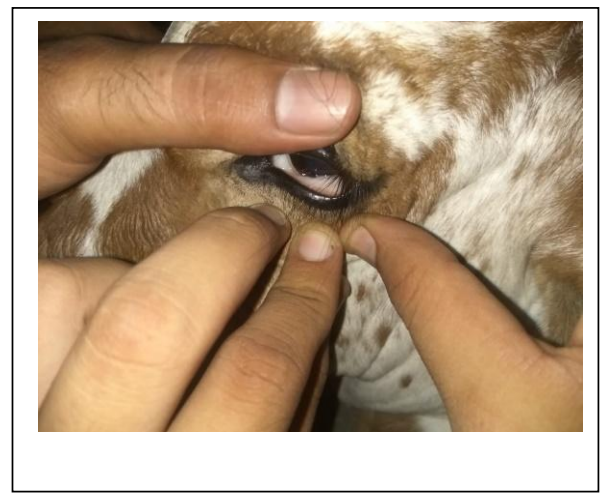


Fig.3 Microscopic examination of blood smear depicted Koach blue body

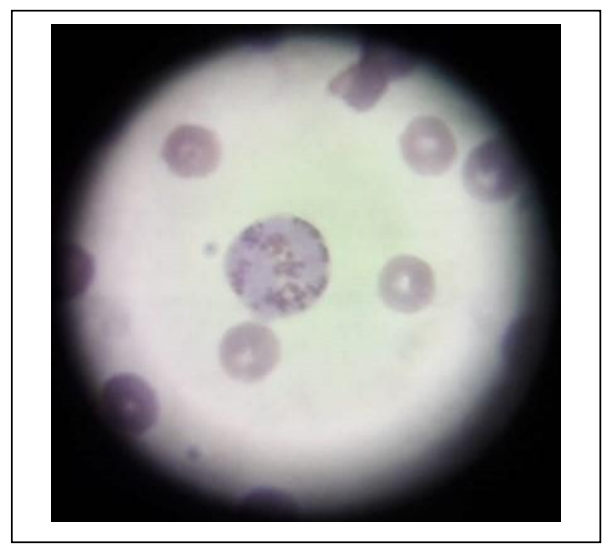

\section{Summary}

Successful treatment of cerebral theileriosis in two gir calves has been reported.

\section{References}

Bagherwal, R. K. 1989. Indian Veterinary Journal, 66: 653.

Dolan, T. T., Injairu, R., Gisemba, F., Main, J.N., Mbwira, S.K., Muela, G.H.M. and Otheeno, D.A.O. 1992. Verinary Rec., 130: 536.
Khanna, B., Dhar, M., and Gautam, O. 1983. Indian veterinary Journal, 60: 603.

Lal, H. and Soni, J. L. 1985. Indian Journal of Animal Sciences, 55: 85.

Morrison, W. I. 1998. theileriosis. In the Merck Veterinary Manual. $8^{\text {th }}$ edn. Ed. S.E. Aiello. Philadelphia, Merck, pp. 31.

Saville, W. L. A. 2002. Ceribral Theileriosis. In large animal internal medicine. $3^{\text {rd }}$ Ed. P.S. Bradford. St. Louis, Mosby, pp. 917.

\section{How to cite this article:}

Bharai, M. J., T. D. Bhavsar, P. G. Dodiya and Vijay L. Parmar. 2019. Therapeutic Management of Theileriosis in Gir Calves: Study of Two Cases. Int.J.Curr.Microbiol.App.Sci. 8(12): 196-198. doi: https://doi.org/10.20546/ijcmas.2019.812.028 